\title{
Castleman's disease
}

\author{
Ashraf A. Kasem, Talal Al Thubaity
}

Department of ENT, Armed Forces Hospitals, Taif Region, Kingdom of Saudi Arabia

Correspondence to Ashraf A. Kasem, MB.Ch B, MS, MRCS (ENT) (Glasg) DO-HNS (Glasg), Armed Forces Hospitals, Taif Region, Kingdom of Saudi Arabia

Tel: +966127336100-2201; fax: 966127330168; E-mail: ashrafkasem68@hotmail.com

Received 09 March 2014

Accepted 11 August 2014

The Egyptian Journal of Otolaryngology 2014, 30:371-373

\section{Background and Aims}

Castleman's disease is a rare disease of lymph node with two identified forms - the hyaline vascular type or angiofollicular type and plasma cell type. It usually presents as localized or systemic lymphadenopathy or even as extra nodal mass and may give rise to several differential diagnoses. Fine needle aspiration cytology (FNAC), as the initial investigation, may be misleading. Excision biopsy usually gives the diagnosis.

\section{Materials and Methods}

We report a case of an 8 year old male child who was Presented with a history of a slowly growing mass in his right side of neck for $3 \mathrm{yrs}$. He Was largely asymptomatic except for occasional discomfort around the mass. His past medical and family history was non-specific. On examination a non-tender, firm mass Was palpated. Initially Clinically suspected to be lymphoma, but was later histologically confirmed to have Castleman's disease.

\section{Result}

We had one case of Castleman's disease which presented as cervical lymphadenopathy of $3{ }^{*} 2 \mathrm{cms}$ in 8 year child. Patient was mostly asymptomatic with all relevant investigations within normal limits. The cytology findings mostly showed a predominance of small lymphocytes with capillary fragments. On excision biopsy, the case was diagnosed as Castleman's disease of the hyaline vascular type.

\section{Conclusion}

Castleman's disease should be kept as a differential of lymphadenopathy, especially in asymptomatic and young patients.

\section{Keywords:}

Castleman's disease, cytology, hyaline vascular type, lymph node, lymphadenopathy

Egypt J Otolaryngol 30:371-373

(c) 2014 The Egyptian Oto - Rhino - Laryngological Society

1012-5574

\section{Introduction}

Castleman's disease (CD) is a rare benign lymphoproliferative disorder that may present as a nodal or an extra nodal mass. Microscopically, two distinct histological patterns have been described - the hyaline vascular (HV) type or angiofollicular type and the plasma cell (PC) type. CD may be confused with other causes of lymphadenopathy. Although CD is a relatively rare entity, it should be strongly considered in the differential diagnosis of cervical lymphadenopathy. In this study, we present a case of $\mathrm{CD}$ of $\mathrm{HV}$ type involving the right cervical lymph node in an 8-year-old boy.

\section{A case report}

An 8-year-old boy was presented with a history of a slowly growing mass on the right side of his neck since 3 years. He was largely asymptomatic except for occasional discomfort around the mass. His past medical and family history was nonspecific. On examination, a nontender, firm mass was palpated but there was no generalized lymphadenopathy or organomegaly. All his blood results were normal, including those for abnormal hormone production. A CT scan revealed evidence of a well-defined, soft tissue mass, seen at the right carotid space. It measured about $3.8 \times 2.2 \times 2 \mathrm{~cm}$ (in $\mathrm{L} \times \mathrm{W} \times$ AP, respectively).It had mild homogenous enhancement with no definite areas of cystic degeneration inside. It was seen mildly compressing the parotid gland anterolaterally and the carotid vessels medially (Fig. 1).

On complementary US, it appeared as a well-defined, oval, soft tissue mass, with rich Doppler color flow inside, which had a main central distribution, these signs mostly suggestive of lymph nodes. Other multiple lesions having the same characteristics but smaller in size were seen at both carotid spaces in the upper and lower neck, posterior triangles, and supraclavicular area, which are suggestive of enlarged cervical lymphadenopathy. There was no specific indication in the laboratory tests and imaging examinations; however, MRI/angiography has potential value on clinical and differential diagnosis.

A surgical excision of the mass was carried out under general anesthesia and the histopathology of the mass showed a hyaline vascular variant of localized CD (Fig. 2). An extended immunostain panel including CD4, CD5, CD20, CD15, CD30, plasma marker, kappa and lambda, and CD68 demonstrated no evidence of clonal lymphoid proliferation. The patient was referred to a hematologist. Tests for HIV and 
Figure 1

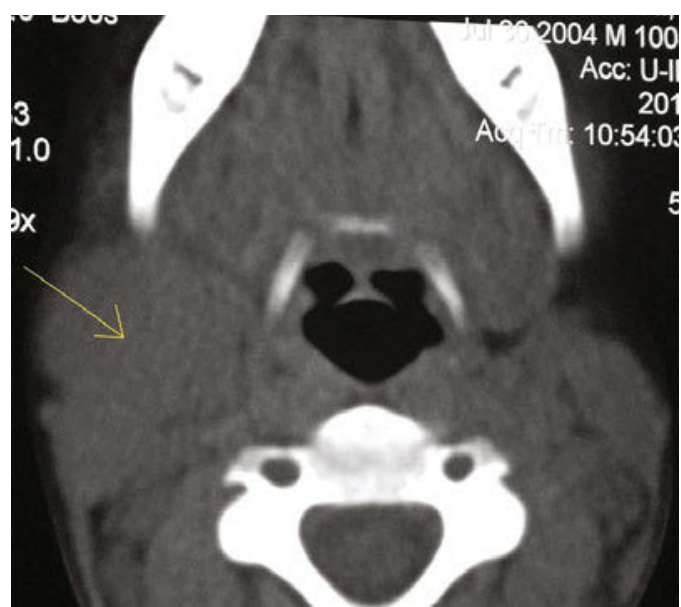

Contrast-enhanced axial CT scan shows an evidence of a welldefined soft tissue mass is seen at the right carotid space. It is seen measuring about $(3.8 \times 2.2 \times 2 \mathrm{~cm})$ in $(\mathrm{L} \times \mathrm{W} \times \mathrm{AP})$ respectively. It has mild homogenous enhancement with no definite areas of cystic degeneration inside. It is seen mildly compressing the parotid gland anterolateral and the carotid vessels medially.

human herpesvirus (HHV8) were carried out, both of which were negative. The patient was asymptomatic 12 months postoperatively with no further lymph node enlargement.

\section{Discussion}

$\mathrm{CD}$ is an uncommon clinicopathological entity characterized by non-neoplastic lymph node hypertrophy and histologically characterized by angiofollicular lymph node hyperplasia [1]. Castleman et al. [1] first described it in 1956 in a group of patients with localized benign lymphadenopathy.

The incidence of CD is not known and can occur at any age, although it has mostly been reported in adults in the literature. The etiology of this disease is unknown; however, it has been found in association with Kaposi's sarcoma. The synonyms are angiofollicular lymph node hyperplasia, angiofollicular lymphoid hyperplasia, giant lymph node hyperplasia, lymphoid hamartoma, and benign lymphoma.

Clinically, the disease has two forms: localized, as first described by Castleman, which is more common; and multicentric disease (MCD), with involvement of several sites, which was first described by GABA et al. in 1978 [2].

Histologically, the main types are the HV type and the PC type. The HV type is characterized by lymphoid follicular proliferation at different levels of maturity, often forming a layered or 'onion-skin' pattern surrounding

\section{Figure 2}

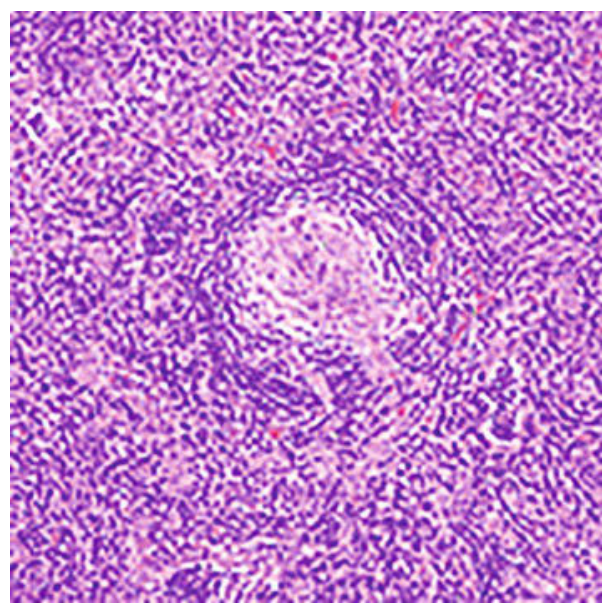

Photomicrograph (original magnification, $\times 100$; hematoxylin and eosin stain) of hyaline -vascular variant of localized Castleman's disease

a hyalinized vessel at the center of the follicle. These vessels are often prominent and reactive. This is more commonly seen in the localized form of the disease.

The $\mathrm{PC}$ variant has sheets of mature $\mathrm{PCs}$ within the interfollicular tissues surrounding larger germinal centers and having significantly less vascularity. The multicentric form of the disease is nearly always associated with this variant. A third histological variant showing a mixed picture can also be seen in MCD.

The localized form of the disease s mostly asymptomatic with a single-site lymph node enlargement. The sites commonly involved are the abdomen, peripheral lymph nodes, and the mediastinum. It is often discovered incidentally during routine examination, chest radiographs, or because of discomfort secondary to local compression, as is in our case described above. Diagnosis is made by histological analysis of the lymph node biopsy to distinguish it from a thymoma.

MCD, however, presents with systemic symptoms along with multiple lymph node hyperplasia. The systemic symptoms are thought to be primarily a consequence of elevated interleukin-6 production. They are present as asthenia, weight loss, fever, and polyadenopathy with a mean of four-site involvement, and are often associated with hepatosplenomegaly.

Some forms of MCD have been found in association with Kaposi's sarcoma, which develops during the clinical course of most HIV-positive MCD cases [3]. An association with HIV-negative Kaposi's sarcoma has also been seen to a much lesser extent. HIV-positive MCD shows an increased prevalence of pulmonary symptoms and can be differentiated from other types of HIV-associated systemic lymphoproliferative 
disorders. There is also an increased progression of HIV-positive MCD to HHV8-associated malignant non-Hodgkin's lymphoma [4].

Routine blood tests usually reveal anemia, elevated ESR, thrombocytopenia, and elevated polyclonal gamma globulins. Identifying an immunophenotypically varied population of B-lymphocytes with polyclonal surface and cytoplasmic immunoglobulin markers helps to confirm the diagnosis of $\mathrm{CD}$ and differentiate it from lymphoma [5]. HIV testing should be carried out with the patient's permission. Herpes virus (HHV8/KSHV) has been isolated in almost all cases of HIV-associated Kaposi's sarcoma MCD and some non-HIV Kaposi's sarcoma MCD, prompting tests for KSHV 6. Histological examination of the biopsied lymph node is essential for the diagnosis. These findings suggest two possibilities concerning the genesis of $\mathrm{CD}$; the first is the opportunistic presence of HHV8, favored by immune perturbations, and the second is the direct pathogenic role of HHV8, in association with dysregulation of cytokines.

Localized CD usually has a good prognosis and requires surgical excision of the enlarged lymph node with no further treatment. The patients generally remain asymptomatic thereafter.

$\mathrm{MCD}$, however, tends to have a variable prognosis with no documented treatment consensus. A variety of combination treatments have been tried with surgical excision, chemotherapy, and steroids. In patients with associated Kaposi'ssarcoma,monthlypolychemotherapy (e.g. cyclophosphamide, vincristine, doxorubicin, and prednisone) has been tried with limited success. AntiIL 6 antibodies have shown success with systemic symptoms, as have steroids. Most treatment modalities involve immunosuppression, increasing the chances of opportunistic infections. Recent suggestions are that treatment with the antiherpesvirus drug ganciclovir or the anti-CD20 B-cell monoclonal antibody, rituximab, may markedly improve outcome $[6,7]$.

\section{Conclusion}

Although the best treatment for CD is still unknown, surgical removal of the localized type of the mass has long been considered a standard therapy for the disease. In our patient, a complete surgical excision was accomplished, and, until now, there is no evidence of recurrence.

\section{Acknowledgements \\ Conflicts of interest}

None declared.

\section{References}

1. Castleman B, Iverson I, Menendez VP. Localized mediastinal lymph node hyperplasia resembling thymoma. Cancer 1956; 9:822-830.

2. Gaba AR, Stein RS, Sweet DL, Variakojis D. Multicentric giant lymph node hyperplasia. Am J Clin Pathol 1978;69:86-90.

3. McCarty MJ, Svetislava JV, Banks PM. Angiofollicular lymph node hyperplasia (Castleman's disease). Cancer Treat Rev 1995; 21:291-310.

4. Talat N, Schulte KM. Castleman's disease: systematic analysis of 416 patients from the literature. Oncologist 2011; 16:1316.

5. Roca B. Castleman's disease. A review. AIDS Rev 2009; 11:3.

6. Casper C. The aetiology and management of Castleman disease at 50 years: translating pathophysiology to patient care. $\mathrm{Br} \mathrm{J}$ Haematol 2005; 129:3.

7. Schulte KM, Talat N. Castleman's disease - a two compartment model of HHV8 infection. Nat Rev Clin Oncol 2010; 7:533. 